\title{
La fiscalité locale : regards comparatifs, simple évolution ou véritable révolution? Propos introductifs
}

\author{
Mots-c|és : fiscalité - fiscalité locale - relations entre l'État et les collectivités territoriales - \\ autonomie financière - autonomie fiscale
}

\begin{abstract}
En France, l'État a recentralisé la fiscalité locale. Les collectivités territoriales françaises ne disposent pas de l'autonomie fiscale. Un regard comparatif et une réflexion sur les effets du numérique pourraient guider les évolutions ; voire les révolutions de notre fiscalité locale.
\end{abstract}

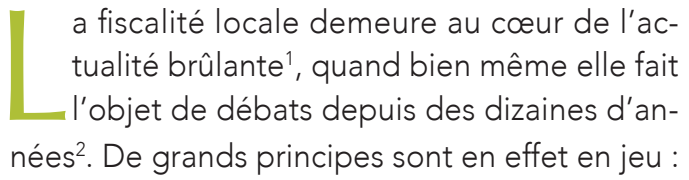

\footnotetext{
I. Feuerstein, M. Quiret, Taxe d'habitation, redevance...: : ce que prévoit la réforme des impôts locaux, Les Echos, 18 juin 2019 ; G. Guichard, La bombe à retardement de la réforme de la fiscalité locale, Le Figaro, 24 septembre 2019

2 L'avènement de la Cinquième République pose les grandes orientations d'une fiscalité locale modernisée (Ord. N 59-108 du 7 janv. 1959 et loi d'application $n^{\circ} 73-1229$ du 31 déc. 1973), mais elle acquiert une réelle importance avec la décentralisation des années 1980. V. dernièrement A. Richard et D. Bur, Mission finances locales, Rapport sur la refonte de la fiscalité locale, mai 2018 ; CESE, Pour une réforme globale de la fiscalité locale, avis 2018-11, avril 2018. Plus anciens, Conseil des impôts, La taxe professionnelle, Quinzième rapport au Président de la République, Imprimerie des Journaux Officiels, 1997

${ }^{3}$ M.-C. Steckel-Montes, Un pouvoir fiscal en trompe-l'œill, Revue française de droit constitutionnel, 2005/1, n 61 , p. $19 \mathrm{~s}$.

${ }^{4}$ Les périmètres fiscaux se sont déjà consolidés avec le développement de l'intercommunalité à fiscalité unique v. généralement, Perspectives d'évolution de la fiscalité locale, Rapport d'information n 289 (2002-2003) de M. J. Arthuis, fait au nom de la commission des finances, déposé le 13 mai 2003 (spéc. p. 199) et disponible sur https://www.senat.fr/rap/r02-289/r02-2891.pdf.

${ }^{5}$ Mais loin d'être inexistante : ainsi, dans le rapport précité, certaines comparaisons étaient présentées (France - Allemagne, France - Canada, ou encore France - Suisse).

${ }^{6}$ OCDE et KIPF, Fiscal Decentralisation and Inclusive Growth, Paris, Editions OCDE, 2018, http://oe.cd/ fiscalnetwork. Cela n'est pas nouveau : voir par exemple dans le rapport précité l'audition de M. Hansjörg Blöchliger, administrateur principal à l'OCDE.

${ }^{7}$ Conseil des prélèvements obligatoires, Fiscalité locale et entreprises, 2014, p. 38, 46, 145.

${ }^{8}$ M. Cozian, F. Deboissy, M. Chadefaux, Précis de fiscalité des entreprises, 2019/2020, n 1853.
}

légalité de l'impôt et égalité devant l'impôt, pouvoir fiscal local et liberté encadrée ${ }^{3}$, réforme fiscalo-fiscale ou territoriale ${ }^{4}$. Leur appréhension par des regards comparatifs n'est en revanche pas toujours systématiquement menée en France ${ }^{5}$. Or si ces expériences étrangères intéressent I'OCDE', ne pourraient-elles pas aussi éclairer la discussion sur la fiscalité locale française? Toutes et tous s'accordent pour dénoncer sa complexité7. Certains n'hésitent pas à parler de vieillerie, voire d'un état de délabrement avancé ${ }^{\text {. }}$ Des illustrations, comme celles de l'octroi de mer ou du "fédéralisme asymétrique », dépeindront comment des « anomalies fiscales » se sont installées durablement dans certains départements et régions françaises d'Outre-mer, mais aussi ailleurs (Pays Basque et Navarre en Espagne). Et en effet, ces exemples et d'autres seront analysés dans les pages qui suivent, offrant les actes les actes du colloque tenu à Lyon fin 2019 sous la direction scientifique de Messaoud Saoudi. 
Et parmi les questions fondamentales qui y ont été posées, I'une des plus essentielles est celle de savoir s'il faut faire simplement évoluer cette fiscalité locale, ou la révolutionner sans détours, notamment dans la perspective de l'économie numérique. Après tout, cette fiscalité largement physiocrate ne reflète ni les richesses d'aujourd'hui, ni celles de demain. Actualité, grands principes, et avenir de la fiscalité locale, tels sont les trois points de ces propos introductifs.

\section{| - Ĺactualité re-centralisatrice française}

Les nouvelles les plus récentes d'abord sont sans doute la suppression de la taxe d'habitation?. Si cette taxe disparaît, comment financer alors les communes? A leur profit serrait réaffectée l'intégralité d'un autre impôt local existant (la taxe foncière) sans création d'un nouvel impôt ${ }^{10}$. Or aujourd'hui cette taxe foncière est aussi en partie perçue par les départements. Ceux-ci se verraient donc amputer d'une ressource dès lors que l'on décide d'affecter la taxe foncière entièrement au bloc communal. L'option adoptée par les députés est de remplacer ces 14 milliards d'euros de taxe foncière des départements par une fraction de la TVA ${ }^{11}$. Le bénéfice d'impôts d'État serait donc partagé entre l'État et les collectivités $^{12}$. Est-ce à dire que la TVA doit être regardée comme un impôt local ? On sait que la définition de la fiscalité locale est claire: son critère est tiré de l'affectation de la recette fiscale. Un impôt est un impôt local lorsque son produit est perçu par une collectivité locale $e^{13}$. Quid d'un impôt à l'origine d'État qui est affecté aux collectivités territoriales ? Cette technique n'est pas nouvelle, mais le phénomène s'amplifie et atteste de la re-centralisation, en France, de la fiscalité locale.

Pire - et cela est antérieur à cette singularité - les impôts traditionnellement locaux sont maintenant décidés au niveau national. Depuis le $1^{\mathrm{er}}$ janvier 2010 la taxe professionnelle a été remplacée par la contribution économique territoriale (CET). Celle-ci repose sur deux assiettes différentes: la première est composée de la cotisation foncière des entreprises (CFE) qui est la reprise de la partie foncière de l'assiette de la taxe professionnelle. Elle donne encore lieu à un vote local de son taux d'imposition, en plus d'avoir une assiette locale - le foncier local. Mais s'agissant de la seconde assiette de la CFE (la cotisation sur la valeur ajoutée des entreprises ou CVAE), celle-ci obéit désormais à un barème national. Cette unicité de taux fixé par l'État est d'ailleurs validée par le Conseil constitutionnel ${ }^{14}$ puisqu'il suffit d'avoir une part locale d'assiette pour que la ressource soit considérée comme une ressource propre de la collectivité locale.

Mais ces actualités, plus ou moins récentes, de la fiscalité locale ne se comprennent bien et ensuite qu'en renouant avec une chronologie inversée. C'est dans les années 30, et plus particulièrement avec la crise économique de 1929 qui force un partage entre l'État et les collectivités territoriales, que l'on date la montée en puissance du pouvoir fiscal local. L'État se voit attribuer les impôts les plus rentables, tandis que les collectivités territoriales récupèrent les anciennes contributions : il leur est alors attribué une fiscalité propre, en leur fournissant la ressource qui est la condition première de l'autonomie locale. Cette autonomie allait s'affirmer dans les années $70,{ }^{15}$ alors même que l'on était toujours en pleine crise, pétrolière cette fois-ci ${ }^{16}$. Cette émancipation se renforce en mars $2003^{17}$ dès lors que pour la garantir, on introduit son principe dans la Constitution $^{18}$.

L'autonomie financière des collectivités locales ne se double pas d'une autonomie fiscale. Une collectivité ne peut pas créer un impôt puisque seule la loi peut autoriser annuellement la perception d'impositions locales. Cela explique la complexité des relations entre l'État et les collectivités territoriales. Cette tension extrêmement forte entre la représentation nationale qui légitimement détient le pouvoir fiscal, et la re-

\footnotetext{
${ }^{9}$ L'article 5 de la loi n²017-1837 du 30 décembre 2017 de finances pour 2018 prévoit la mise en place d'un dégrèvement de taxe d'habitation sur la résidence principale selon les revenus, ce dégrèvement sera progressif sur trois ans. A l'horizon 2020, 80 \% de la population sera dégrevée de TH, ceci devrait être neutre puisque l'État compensera les collectivités territoriales afin de ne pas pénaliser leurs ressources. V. J. Dussel, Ressources des collectivités locales : fiscalité, emprunts et gestion de la dette, JCl. Collectivités territoriales, fasc. 2800 (28 juin 2019); dans son prolongement, le projet de loi de finances pour 2020 prévoit la suppression progressive mais totale et définitive de la taxe d'habitation sur les résidences principales.

${ }^{10}$ Projet $n^{\circ} 2272$, enregistré à la Présidence de l'Assemblée nationale le 27 septembre 2019, disponible sur http:// www.assemblee-nationale.fr/15/projets/pl2272.asp.

${ }^{1}$ Afigese, Réforme fiscale : alerte sur les conséquences financières pour les départements du remplacement de la taxe foncière départementale par une fraction de TVA nationale, 5 août 2019, disponible sur https:// afigese.fr/2019/08/05/reforme-fiscale-alerte-sur-les-consequences-financieres-pour-les-departements-duremplacement-de-la-taxe-fonciere-departementale-par-une-fraction-de-tva-nationale/.

${ }^{12}$ Ceci est vrai pour la TVA, mais aussi pour la taxe intérieure de consommation sur les produits énergétiques - la TICPE.

${ }^{13}$ Pour un résumé, voir les conclusions de R. Victor sous CE, 8e et 3e ss-sect., 29 mai 2017, n 391930, SAS Campeli : Rev. dr. fisc. 2017, nº 41 comm. 497, § 3

${ }^{14}$ CE, 9e et 10 e ss-sect., 31 juill. 2009, n 304109, Fillatre : RJF 2009, n 1098 ; BJDU 5/2009, p. 381, concl. C. Legras ; CE, 9 e et 10 e ss-sect., 20 mars 2013, n 345317, Otto-Bruc: RJF 6/2013, nº 619.

${ }^{15} V_{\text {. supra note }}{ }^{\circ}$ 2. V. la loi $n^{\circ} 75-678$ du 29 juillet 1975 créant la taxe professionnelle pour mettre fin à une imposition jugée antiéconomique : J. Lamarque, O. Négrin, L. Ayrault, Droit fiscal général, $4^{\circ}$ éd. 2016, nº 1924.

${ }^{16}$ Toutes les crises n'ont pas toujours les mêmes effets en matière de fiscalité locale. La dernière crise pétrolière a conduit l'Algérie à entamer une réflexion le renforcement de sa fiscalité locale au détriment du rôle de l'État: Les collectivité territoriales : acteurs du développement local dans les pays du Maghreb, Université d'Annaba, 2017, et la contribution de G. Cavalier, De quelques solutions françaises pour une meilleure gestion des finances des collectivités locales, et résumée dans le journal Seybouse times du 15 oct. 2017.

${ }^{17}$ V. généralement, J.-L. Albert, Finances publiques, 11e éd., Dalloz (2019), $n^{\circ} 42 \mathrm{~s}$

${ }^{18}$ Art. $72-2$
} 
O s s i er

$>$ La fiscalité locale : regards comparatifs Colloque de Lyon 10 octobre 2019

\begin{abstract}
${ }^{19}$ La Vénétie, la Lombardie et l'Emilie-Romagne représentent environ $40 \%$ du PIB italien dans le pilotage du système financier territorial, ${ }^{21}$ lequel annonce peut-être aussi une certaine responsabilisation financière.
\end{abstract}

${ }^{20}$ La Catalogne recherche ce type de statut pour réduire sa participation au système de péréquation.

${ }^{21}$ V. Dussart, La renationalisation de la fiscalité locale, REIDF 2019/1, p. 41, et plus généralement l'important dossier de cette revue intitulé "Le fédéralisme et le régionalisme fiscal».

22 V. notamment M. Bouvier Le Conseil constitutionne et l'autonomie fiscale des collectivités territoriales : du quiproquo à la clarification, Nouveaux cahiers du Consei Constitutionnel $n^{\circ} 33$, oct. 2011.

${ }^{23} \mathrm{~J}$.-L. Albert, préc. $n^{\circ} 715$.

${ }^{24} \mathrm{~V}$. le compte rendu de la séance du mardi 21 déc. 1965 JORF, débats AN, $n^{\circ} 103$, en date du 22 déc. 1965

${ }^{25}$ Y. Fréville, Les finances des collectivités locales au début des années 1960 Une approche statistique au niveau des grandes villes de province, Economies et finances, Thèse Rennes I, 1966, not. p. 219.

${ }^{26} \mathrm{~J}$.-L. Albert, préc. $n^{\circ} 716$. ${ }^{27}$ Id. $n^{\circ} 717$.

${ }^{28}$ Id. $n^{\circ} 719$

${ }^{29} \mathrm{~V}$. par ex. les compte-rendus des séances des mercredi 23 et jeudi 24 juin 1965 , JORF, débats AN, $n^{\circ} 52$ en date des 24 et 25 juin 1965 (p. 2434 dans l'édition publiée le 25 juin); v. encore J.-P. Dussaife, Le Parlement face à la réforme des taxes sur le chiffre d'affaires, Revue française de science politique 1966, p. 521.

30 J.-L. Albert, préc. $n^{\circ} 723$. Autrement dit, à côté de la péréquation verticale, ou descendante (les transferts de l'État vers les collectivités), on trouve aussi une péréquation horizontale, qui s'effectue entre collectivités. En effet, certains mécanismes de péréquation sont inclus dans la DGF.

${ }^{31}$ H. Blöchliger, C. Charbit, Péréquation financière Rev. éco. de l'OCDE 2008/1 $\left(n^{\circ} 44\right)$, p. 283 présentation territoriale qui doit répondre à des besoins de plus en plus importants, s'est révélée lors de la troisième et dernière crise financière et économique, et pas seulement en France. Cette dernière a replacé l'État français au premier plan. L'Italie a elle-aussi dû faire une pause dans son "fédéralisme fiscal » alors que le nombre de régions voulant disposer d'un statut particulier s'accroit, ${ }^{19}$ tout comme ailleurs en Europe ${ }^{20}$. Cette tendance qui s'exprime sera analysée à travers le prisme du renforcement du rôle de l'État

Si l'on s'accorde sur ce constat, une interrogation demeure : quels sont les motifs ayant mené en France au passage d'une fiscalité locale forte à une autonomie fiscale locale sur le déclin? Peut-on y voir l'influence de l'Europe et le respect du pacte de stabilité et de croissance? Ou la véritable raison doit-elle être recherchée ailleurs ? Peut-être réside-t-elle tout simplement dans l'évolution de nos règles ayant conduit à des choix fiscaux aboutissant à miner le système fiscal local de l'intérieur : allègement des impôts sur les entreprises, en particulier l'ancienne taxe professionnelle, dégrèvements, exonérations, de telle sorte qu'une partie de la fiscalité locale a eu tendance à disparaître progressivement. ${ }^{22}$ L'État a donc dû procéder à des compensations, car la fiscalité locale, qui s'inscrit dans des théories de finances publiques, doit composer avec de grands principes.

\section{II - Les grands principes en finances publiques comparées}

Ces grands principes, au premier rang desquels ceux d'égalité et de justice fiscale, imposent à l'État de reverser aux collectivités locales le manque-à-gagner résultant des allégements évoqués ci-dessus. Ces compensations, qui se sont progressivement transformées en dotation, aboutissent d'abord à des transferts verticaux, de I'État vers les collectivités. Ils représentent une part essentielle des budgets locaux - environ 30 \%. Pour 2019, la loi de finances fait apparaître une affectation d'environ 110 milliards d'euros de transferts de ressources d'État au profit des collectivités ${ }^{23}$. Ils prennent trois formes:

la dotation globale de fonctionnement (DGF) qui représente - toujours en 2019 - près de $56 \%$ de ces 110 milliards d'euros. Elle illustre notamment la volonté de préserver l'égalité entre les communes. II s'agit d'une idée ancienne dont on discute déjà en 1965, au moment où l'on rem- place une taxe locale par une taxe d'État ${ }^{24}$. En effet, lorsqu'on instaure l'impôt d'État qu'est la TVA, on supprime la taxe locale qui frappait les commerçants détaillants et les artisans. Or cette taxe locale apporte des ressources aux collectivités locales - près de la moitié des ressources fiscales des communes dans les années 196025. Pour la remplacer dans leurs budgets, cette dotation globale de fonctionnement est mise en place en 1979 et représente à l'époque et déjà plus de la moitié des concours financiers de l'État aux collectivités ${ }^{26}$.

C'est donc à l'occasion d'une mesure purement technique - la généralisation de la TVA au commerce de détail - que l'on observe une première transformation radicale du financement des collectivités.

La deuxième forme de fiscalité transférée est constituée par les dotations et subventions d'équipement. Leurs montants sont beaucoup plus faibles que la DGF - un dixième des transferts de l'État ${ }^{27}$ - et ces dotations comprennent le fonds de compensation pour la TVA (FCTVA). Sa fonction est de « restituer » la TVA que les collectivités locales paient à l'État à l'occasion de leurs investissements qui supporte effectivement la taxe.

La troisième forme de transferts de l'État est constituée par les dotations de compensation, lesquelles sont cette fois-ci plus importantes que la DGF : elles représentent la moitié de ces transferts. ${ }^{28}$ Plus précisément, elles sont la contrepartie des exonérations et dégrèvements législatifs que l'État accorde à certains contribuables locaux.

Ce principe d'égalité entre l'État et ses collectivités territoriales se décline aussi et ensuite entre les collectivités locales elles-mêmes. Leur situation financière est en effet marqué, de manière ancienne et connue, par de fortes inégalités. La taxe locale frappant les commerçants détaillants et les artisans jusqu'en 1965 crée de très grandes inégalités : les centres commerciaux bénéficient d'attributions directes souvent exagérées alors que celles des villes-dortoirs ou des communes rurales se trouvent réduites au minimum garanti ${ }^{29}$. Toute augmentation de l'autonomie locale accroit ces inégalités.

Pour réduire ces écarts de richesse, le législateur a donc développé d'autres mécanismes dits de péréquation. ${ }^{30}$ En matière financière, la péréquation est ce « transfert de ressources budgétaires entre les différentes collectivités territoriales dans le but de compenser les différences dans la capacité de prélèvement fiscal ou dans le coût des services publics $»^{31}$. La redistribution permet 
donc d'accommoder le principe d'égalité à celui de justice fiscale.

Entre collectivités locales, ces mécanismes existent aux niveaux régional et local et sont passablement compliqués. Surtout si l'on songe à la forme la plus aboutie d'intercommunalité que sont les établissements publics de coopération intercommunale (EPCI) pouvant avoir une fiscalité propre (e.g. métropole, communauté urbaine, communauté d'agglomérations et communautés de communes) ${ }^{32}$. Ils donnent lieu à des litiges classiques entre personnes publiques dont un intervenant aura l'occasion de dresser une typologie. Ces contentieux se retrouvent dans la tentation de certaines collectivités de voter des taux excédentaires ou encore d'assujettir des professionnels qui n'auraient pas normalement dû l'être ${ }^{33}$ : cela pose en palimpseste l'avenir de certaines taxes, comme celle d'enlèvement des ordures ménagères ${ }^{34}$ (TEOM) qui, elle aussi, sera abordée. Ces tensions s'expliquent aussi en partie par l'injection de droit souple dans la solidarité financière intercommunale.

Il sera alors intéressant d'examiner si cette démarche de solidarité territoriale est une particularité française. Car l'application de ces grands principes mérite d'être examinée sous des regards comparatifs au pluriel. Cette mise sous tension européenne de la fiscalité locale nous apprendra sans doute que ce mouvement de recentralisation s'observe ailleurs en Europe. L'Allemagne, I'Espagne ${ }^{35}$, I'Italie, ou la Suisse, sont à cet égard intéressants : pour se limiter à la Constitution italienne, celle-ci prévoit elle-même un fonds de péréquation établi par l'État ${ }^{36}$.

Au-delà de cette mosaïque proposant des reflets communs, la question fondamentale à laquelle le présent dossier aimerait apporter sa contribution est la suivante : faut-il souhaiter une révolution de la fiscalité locale?

\section{|II - Évolution ou révolution?}

La fiscalité locale est-elle ce simple fruit d'une évolution, ou de nouveaux contextes appellent-ils une véritable révolution? Compte tenu de son actualité, et du sempiternel constat de délabrement qui l'affecte, la fiscalité locale a certes besoin d'être simplifiée et modernisée. Elle a aussi besoin d'être mieux répartie également, ce qui n'est pas chose aisée dans un pays qui compte quelques 36000 communes $^{37}$.

On répète que le calcul de la valeur locative est vieux et compliqué. Les mécanismes d'imposition utilisent des techniques de recouvrement d'un autre âge ${ }^{38}$. Et mêmes une imposition locale récemment réformée (la $C F E$ ) présente un décalage de deux ans entre la détermination de la base et le paiement de l'impôt ${ }^{39}$. De cette façon, une entreprise qui vend un immeuble en 2019 paiera deux ans plus tard (en 2021) un impôt sur un bien dont elle n'a plus la disposition.

Est-ce que la blockchain pourrait permettre de réduire cet écart dans le temps en favorisant une actualisation en temps réel et une transmission sécurisée des informations? La Charte européenne des droits de l'homme dans la ville, dont la ville de Lyon est signataire, prévoit que les autorités locales assurent la transparence et le contrôle rigoureux de l'usage des fonds publics ${ }^{40}$. L'utilisation de ces technologies pourrait-elle permettre une meilleure transparence et un contrôle accru? Certes les informations nécessaires à la fiscalité locale sont centralisées, d'une manière ou d'une autre: mais certains États, comme la Finlande ${ }^{41}$ ou encore l'Estonie ${ }^{42}$ sont bien plus avancés dans leurs réflexions, et des projets ont même permis de modéliser l'utilisation de ces technologies en matière de fiscalité locale ${ }^{43}$.

Et au-delà de la détermination de l'impôt, ne faut-il pas imaginer de nouvelles bases pour la fiscalité locale, dépassant la seule richesse immobilière ? Cette richesse du XXVIII e siècle n'est plus celle d'aujourd'hui : le capital est désormais de plus en plus numérique. Avec l'amélioration des données et des systèmes, de nouveaux choix de politiques financières s'offrent aux décideurs. ${ }^{44}$

\footnotetext{
${ }^{32} \mathrm{Au} 1$ er janvier 2019, on recense 1258 établissements publics de coopération intercommunale (EPCI) à fiscalité propre sur le territoire français. Parmi ceux-ci, on dénombre 21 métropoles, 13 communautés urbaines, 223 communautés d'agglomération et 1001 communautés de communes : v. Cartographie des EPCl à fiscalité propre disponible sur https://www.collectivites-locales.gouv.fr/cartographie-des-epci-a-fiscalite-propre.

${ }^{33} \mathrm{CE}, 31$ mars 2014, 8e-3e s.-s., Ministre Budget c/ Société Auchan France, $n^{\circ} 368111$.

${ }^{34} \mathrm{CGl}$, art. 1522

${ }^{35}$ Const. espagnole, art. 156

${ }^{36}$ Const. italienne, art. 119 ; sur cet article, dont l'entrée en viqueur a été empêché, v. A. Deffenu, Le fédéralisme fiscal en Italie : I'article 119 de la Constitution entre crise économique et transition infinie, REIDF 2019/1, p. 87 .

${ }^{37}$ M. Cozian, F. Deboissy, M. Chadefaux, préc., 2019-2020, n 1853. En l'espace de cinquante ans, ce nombre de communes a pourtant été réduit de 2000

${ }^{38}$ Celui-ci s'effectue par voie de rôle.

${ }^{39}$ Cet écart est dû à des nécessités pratiques : I'administration doit disposer de tous les éléments pour calculer l'impôt, notamment les taux, qui sont fixés par les communes.

${ }^{40}$ Art. XXIV-4, dsponible sur https://www.uclg-cisdp.org/fr/activities/villes-pour-les-droits-humains/charteeuropeenne.

${ }^{41}$ En Finlande, les autorités fiscales ont commencé à travailler avec les banques sur un système blockchain pour asssurer le suivi fiscal des transactions immobilières dès 2020. La Finlande a également mené un projet de " proof-of-concept » ou un projet pilote d'évaluation de l'efficacité d'un système de TVA basé sur la blockchain.

${ }^{42}$ Estonia - the Digital Republic Secured by Blockchain, p. 7 (v. https://www.uclg-cisdp.org/fr/activities/villespour-les-droits-humains/charte-europeenne).

${ }^{43}$ V. Blockchain for Municipal tax: http://www.iaacblog.com/programs/blockchain-4-municipal-tax/.

${ }^{44}$ S. Gupta, M. Keen, A. Shah, G. Verdier, Digital Revolutions in Public Finance, IMF, 2017.
} 
Dos sier

$>$ La fiscalité locale : regards comparatifs Colloque de Lyon 10 octobre 2019
Le nom révolution, vient du latin revolvo, qui signifie étymologiquement « rouler en arrière ${ }^{45}$. Dans l'usage courant, il désigne un changement brutal, effaçant la notion de retour.

Si l'on peut s'attendre à ce que le mouvement de recentralisation implique un retour en arrière, le contexte européen et numérique pourrait bien expliquer des changements plus soudains en matière de fiscalité locale. Et évoquer en France une révolution, pourrait rendre le lecteur impatient de parcourir le présent dossier : en France en effet, on aime - peut-être un peu trop ? - les révolutions.

\footnotetext{
5 II s'agit historiquement d'un retour périodique d'un astre à un point de son orbite : v. Dictionnaire historique de la

langue française (dir. A. Rey), Dictionnaire Le Robert (Paris), 1992, p. 1800, Ve Révolution.
} 